\title{
FINITENESS THEOREMS FOR APPROXIMATE FIBRATIONS
}

BY

\author{
D. S. CORAM AND P. F. DUVALL, JR. ${ }^{1}$
}

\begin{abstract}
This paper concerns conditions on the point inverses of a mapping between manifolds which insure that it is an approximate fibration almost everywhere. The primary condition is $\pi_{i}$-movability, which says roughly that nearby point inverses include isomorphically on the $i$ th shape group into a mutual neighborhood. Suppose $f: M^{m} \rightarrow N^{n}$ is a $U V^{1}$ mapping which is $\pi_{i}$-movable for $i<k-1$, and $n>k+1$. An earlier paper proved that $f$ is an approximate fibration when $m \leqslant 2 k-1$. If instead $m=2 k$, this paper proves that there is a locally finite set $S \subset N$ such that $f \mid f^{-1}(N-S)$ is an approximate fibration. Also if $m=2 k+1$ and all of the point inverses are FANR's with the same shape, then there is a locally finite set $E \subset N$ such that $f \mid f^{-1}(N-E)$ is an approximate fibration.
\end{abstract}

1. Introduction. This paper is concerned with conditions on the point inverses of a map which imply that the map is an approximate fibration. In [C-D $\mathbf{3}$, it was shown that if $f: M^{m} \rightarrow N^{n}$ is a $U V^{1}$ mapping which is $\pi_{i}$-movable for $i$ up to the middle dimension, then $f$ is an approximate fibration (terminology will be explained later in this section). In this paper we study the consequences of relaxing the movability assumptions by one dimension and obtain results analogous to Lacher's finiteness theorems $\left[\mathbf{L}_{2}\right],\left[\mathbf{L}_{3}\right]$. As in Lacher's work, there is an even- and an odd-dimensional case. Here are the statements of our main results in this direction.

THEOREM 2.5. Let $f: M^{2 k} \rightarrow N^{n}$ be a mapping between manifolds with $n \geqslant k+1$. If $f$ is $U V^{1}$ and $\pi_{i}$-movable for all $i \leqslant k-1$, then there is a locally finite set $S \subset N$ such that $f$ is an approximate fibration over $N-S$.

THEOREM 4.4. Let $f: M^{2 k+1} \rightarrow N^{n}$ be a $U V^{1}$ mapping between manifolds, $n>k+$ 1. If $f$ is $\pi_{i}$-movable for all $i \leqslant k-1$ and all the point inverses of $f$ have the same shape and are FANR's, then there is a locally finite set $E \subset N$ such that $f$ is an approximate fibration over $N-E$.

The paper is organized as follows. In $\$ 2$ we derive some algebraic information concerning the point inverses of our mappings and prove 2.5 . $\$ 3$ is devoted to a generalization of the winding function analysis used in $\left[\mathbf{C}-\mathbf{D}_{3}\right]$ and $\left[\mathbf{C}-\mathbf{D}_{4}\right]$. $\$ 4$ contains the proof of Theorem 4.4. In $\S 5$ we use the results of this paper to remove

Received by the editors September 17, 1978.

1980 Mathematics Subject Classification. Primary 54C10; Secondary 57N15, 55R65, 57N25.

Key words and phrases. Approximate fibration, movability, mapping between manifolds.

${ }^{1}$ Research of both authors supported by an NSF contract. 
the nondegeneracy assumptions in $\left[\mathbf{C}-\mathbf{D}_{\mathbf{3}}\right]$ and $\left[\mathbf{C}-\mathbf{D}_{\mathbf{4}}\right]$. Finally, in $\S 6$, we give several examples of mappings which illustrate the need for some of the hypotheses in our results.

We shall use the following notations and conventions. We use $H^{i}$ and $H_{i}$ for Čech cohomology and homology with $Z$ ( = integer) coefficients and $\check{\pi}_{i}$ for "Čech homotopy", i.e., $\check{\pi}_{i}(X)$ is the inverse limit of the usual homotopy groups of a neighborhood system of $X$ when $X$ is embedded in an ANR. The worst spaces we shall encounter in homological calculations will be (pointed) FANR's [B], [M]. Since the inverse systems associated with such spaces satisfy the Mittag-Leffler condition [0], we shall use tools such as the Mayer-Vietoris sequence and Universal Coefficient Theorems without further justification.

If $f: E \rightarrow B$ is a mapping, then $F_{b}$ denotes $f^{-1}(b)$ for $b \in B$, and $\tilde{U}$ denotes $f^{-1}(U)$ for $U \subset B$. We study inclusion maps so frequently that we adopt the following convention. Every unlabeled arrow or arrow labeled without explanation indicates an inclusion or inclusion induced homomorphism.

Let $f: E \rightarrow B$ be a proper, surjective mapping between locally compact, separable metric ANR's. We say that $f$ is $\pi_{k}$-movable at $b \in B$ provided that for each neighborhood $U_{0}$ of $F_{b}$ there are open sets $U$ and $V$ with $b \in V \subset U \subset U_{0}$ such that for each $c \in V$ and each $e \in F_{c}, \check{\pi}_{k}\left(F_{c}, e\right)$ maps isomorphically onto the image of $\pi_{k}(\tilde{V}, e)$ in $\pi_{k}(\tilde{U}, e)$ under the inclusion induced homomorphisms. A map is $\pi_{k}$-movable if it is $\pi_{k}$-movable at every point of the range. The property $H_{k}$-movable is defined similarly using homology. Notice that a $k-U V$ mapping [ $\left.\mathbf{L}_{s}\right]$ is $\pi_{k}$-movable and a $k-u v$ mapping [ $\mathbf{L}_{5}$ ] is $H_{k}$-movable. For definitions and basic properties of approximate fibrations, see $\left[\mathbf{C}-\mathbf{D}_{\mathbf{1}}\right]$ and $\left[\mathbf{C}-\mathbf{D}_{\mathbf{2}}\right]$.

We will need the following converse to $\left[\mathbf{C}-\mathbf{D}_{5}\right.$, Theorem $\left.\mathrm{A}\right]$ in $\$ 4$.

LEMMA 1.1. Let $p: E \rightarrow B$ be a mapping between locally compact, separable ANR's. If $p$ is $U V^{1}$ and $\pi_{i}$-movable for all $i \leqslant k$ where $k \geqslant 1$, then $p$ is $H_{i}$-movable for all $i \leqslant k$.

Proof. Without loss of generality we may assume that $E$ and $B$ are $Q$-manifolds [C-D, Lemma 2.2]. Given $b \in B$ and a neighborhood $U_{0}$ of $b$, choose contractible open neighborhoods $V \subset U$ of $b$ in $U_{0}$ satisfying $\pi_{i}$-movability for all $i<k$. Given $c \in V$, choose contractible open neighborhoods $X \subset W$ of $c$ in $V$ again satisfying $\pi_{i}$-movability, $i \leqslant k$.

Now consider the following commutative diagram:

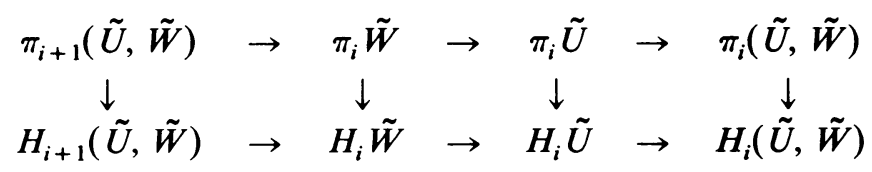

where the horizontal rows are portions of exact sequences of the pair $(\tilde{U}, \tilde{W})$ and the vertical arrows are Hurewicz homomorphisms. When $i<k-1, \pi_{i+1}(\tilde{U}, \tilde{W})=$ $\pi_{i}(\tilde{U}, \tilde{W})=0$ by $\left[\mathbf{C}-D_{5}\right.$, Lemma 2.3$]$. Hence $H_{i+1}(\tilde{U}, \tilde{W})=H_{i}(\tilde{U}, \tilde{W})=0$ and $H_{i} \tilde{W} \rightarrow H_{i} \tilde{U}$ is an isomorphism. When $i=k$, this argument shows only that 
$H_{k} \tilde{W} \rightarrow H_{k} \tilde{U}$ is an epimorphism. To show it is also monic consider that $\pi_{k} \tilde{V} \rightarrow$ $\pi_{k} \tilde{U}$ is likewise an epimorphism. However, $\check{\pi}_{k} F_{c}$ includes isomorphically onto image of $\pi_{k} \tilde{V}$ in $\pi_{k} \tilde{U}$, so $\check{\pi}_{k} F_{c} \rightarrow \pi_{k} \tilde{U}$ must be an isomorphism. Similarly, $\check{\pi}_{k} F_{c} \rightarrow$ $\pi_{k} \tilde{W}$ is an isomorphism. Therefore $\pi_{k} \tilde{W} \rightarrow \pi_{k} \tilde{U}$ is an isomorphism. Also since $p$ is $U V^{1}, \tilde{U}$ and $\tilde{W}$ are simply connected by $\left[\mathbf{L}_{1}\right.$, Lemma 5.1]. Hence $\pi_{k+1}(\tilde{U}, \tilde{W}) \rightarrow$ $\boldsymbol{H}_{k+1}(\tilde{U}, \tilde{W})$ is an isomorphism. A simple diagram chase now shows that $\boldsymbol{H}_{\boldsymbol{k}} \tilde{W} \rightarrow$ $H_{k} \tilde{U}$ is monic. It follows that $H_{i} F_{c} \rightarrow H_{i} \tilde{U}$ is an isomorphism for all $i<k$, so $p$ is $H_{k}$-movable.

2. The even-dimensional case. We first recall a lemma which we shall use frequently.

LEMMA 2.1 [C-D , LeMma 4.1]. If $f: M^{m} \rightarrow N^{n}$ is a $U V^{1}, \pi_{i}$-movable mapping between manifolds for all $i \leqslant k-1$, then for each $y \in N, H^{j}\left(F_{y}\right)=0$ for $j>$ $\max \{m-k+1, m-n+1\}$.

Next we study the cohomology groups of the fiber $F_{y}$ in the next smaller dimension. The following three results are based on an argument in [L-M].

LEMMA 2.2. Let $f: M^{m} \rightarrow N^{n}$ be a mapping between manifolds which is $U V^{1}$ and $\pi_{i}$-movable for all $i \leqslant k-1$, where $n \geqslant k+1$. Suppose that $Y \subset B \subset W \subset N$ where $W$ is homomorphic to $R^{n}, B$ is a tame $n$-cell in $W$ and $Y$ is closed. If $H^{i}(Y)=0$ for $i \geqslant n-k$ and $W-Y$ is simply connected, then the inclusion $H^{m-k} f^{-1}(B) \rightarrow H^{m-k} f^{-1}(Y)$ is an epimorphism.

Proof. $\tilde{W}$ is simply connected since $f$ is $1-U V$, so $\tilde{W}$ is orientable. By duality, $H_{j}(W, W-Y) \cong H^{n-j} Y \cong 0$ for $j \leqslant k$ and $H_{j}(W, W-B) \cong H^{n-j}(B) \cong 0$ for $j<k-1$. It follows from the exact sequence of $(W, W-Y, W-B)$ that $H_{j}(W-Y, W-B)=0$ for $j<k-1$ so that $(W-Y, W-B)$ is $(k-1)$ connected by the relative Hurewicz theorem.

Therefore $(\tilde{W}-\tilde{Y}, \tilde{W}-\tilde{B})$ is $(k-1)$-connected by [C-D, Lemma 2.3] and $H_{k}(\tilde{W}-\tilde{Y}, \tilde{W}-\tilde{B})=0$. Now consider the commutative diagram

$$
\begin{aligned}
& H^{m-k} \tilde{B} \quad \stackrel{\alpha}{\rightarrow} \quad H^{m-k} \tilde{Y} \\
& \downarrow \cong \quad \downarrow \cong \\
& H_{k}(\tilde{W}, \tilde{W}-\tilde{B}) \quad \rightarrow \quad H_{k}(\tilde{W}, \tilde{W}-\tilde{Y}) \rightarrow H_{k-1}(\tilde{W}-\tilde{Y}, \tilde{W}-\tilde{B})
\end{aligned}
$$

where the top row is inclusion, the vertical arrows are duality isomorphisms, and the bottom row is from the sequence of the triple. Since $H_{k-1}(\tilde{W}-\tilde{Y}, \tilde{W}-\tilde{B})=$ $0, \alpha$ is an epimorphism.

LEMMA 2.3. Let $f: M^{m} \rightarrow N^{n}$ be a $U V^{1}, \pi_{i}$-movable mapping between manifolds for all $i \leqslant k-1, n \geqslant k+1$. Suppose that $W$ is a Euclidean neighborhood of a point $y \in N$ and $B$ is a tame $n$-cell neighborhood of $y$ in $W$. Then $H^{m-k} f^{-1}(B)$ is finitely generated.

Proof. Choose an $n$-cell $B_{1} \subset W$ containing $B$ in its interior $V$. Since $\tilde{B}$ is compact and $\tilde{V}$ is an ENR, Lemma 2.1 of $[\mathbf{L}-\mathbf{M}]$ gives a finite polyhedron $P$ and 
maps $\tilde{B} \stackrel{\alpha}{\rightarrow} P \stackrel{\beta}{\rightarrow} \tilde{V}$ such that $\beta \alpha$ is the inclusion. By the above lemma with $Y=B$ and $B=B_{1}, H^{m-k} \tilde{B}_{1} \rightarrow H^{m-k} \tilde{B}$ is an epimorphism. Since this epimorphism factors through $H^{m-k}(P), H^{m-k} \tilde{B}$ is finitely generated.

THEOREM 2.4. If $f: M^{m} \rightarrow N^{n}$ is a $U V^{1}, \pi_{i}$-movable mapping between manifolds for all $i \leqslant k-1$, where $n \geqslant k+1$, then

$$
S=\left\{y \in N \mid H^{m-k} F_{y} \neq 0\right\}
$$

is locally finite.

Proof. Let $y \in N$ and suppose that $y$ is a limit point of $S$. Let $Y$ be $y$ together with a sequence in $S$ converging to $y$. Let $W$ be a Euclidean neighborhood of $y$ and let $B$ be a tame $n$-cell neighborhood of $y$ in $W$. We may assume $Y \subset B$. By the above lemmas $H^{m-k} \tilde{B} \rightarrow H^{m-k} \tilde{Y}$ is epic and $H^{m-k} \tilde{B}$ is finitely generated, so $H^{m-k} \tilde{Y}$ is finitely generated. This is a contradiction since $\tilde{Y}$ has infinitely many separated components with nontrivial cohomology.

THEOREM 2.5. Let $f: M^{2 k} \rightarrow N^{n}$ be a mapping between manifolds with $n \geqslant k+1$. If $f$ is $U V^{1}$ and $\pi_{i}$-movable for all $i \leqslant k-1$, then there is a locally finite set $S \subset N$ such that $f$ is an approximate fibration over $N-S$.

Proof. By Lemma 2.1, $H^{j}\left(F_{y}\right)=0$ for all $j \geqslant k+1$ and all $y \in N$. The set $S=\left\{y \in N \mid H^{k} F_{y} \neq 0\right\}$ is locally finite by Theorem 2.4. If $y \in N-S$, then $F_{y}$ is $i$ - $u v$ for all $i \geqslant k$ by [ $\mathbf{L}_{1}$, Theorem 3.1]. Hence by [C-D $], f \mid \widehat{N-S}$ is $\pi_{i}$-movable for all $i$ and is an approximate fibration [C-D $\left.\mathbf{D}_{2}\right]$.

COROllary 2.6. Let $f: M^{2 k} \rightarrow N^{n}$ be a $U V^{1}$ mapping between manifolds, $n \geqslant k+$ 1. If $f$ is $\pi_{i}$-movable for $i \leqslant k-1$ and all of the point inverses have the same shape, then $f$ is an approximate fibration.

3. Winding functions. Throughout this section suppose that $f: E \rightarrow B$ is a proper surjective mapping between ANR's such that for each $b \in B, F_{b}$ is a FANR. Also suppose that for some $k$ and $q, H_{k}\left(F_{b}\right) \cong Z^{q}$ for all $b \in B$.

Given $b \in B$, one can use the strong movability properties of FANR's [M] to show

(i) if $U$ is a sufficiently small neighborhood of $b, H_{k}\left(F_{b}\right) \rightarrow H_{k}(\tilde{U})$ is monic, and

(ii) if $U$ is any neighborhood of $b$ and $V$ is a sufficiently small neighborhood of $b$ in $U$, there is a homomorphism $r: H_{k}(\tilde{V}) \rightarrow H_{k}\left(F_{b}\right)$ such that

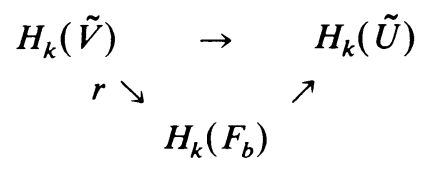

commutes.

If $A$ is a closed subset of $B$, let $K(A)$ be the set of points $b \in A$ such that for each neighborhood $V$ of $b$ there is a point $c \in V \cap A$ such that $H_{k}\left(F_{c}\right) \rightarrow H_{k}(\tilde{V})$ is not monic. $K(A)$ is clearly closed. In fact, we have the following.

LEMMA 3.1. $K(A)$ is closed and nowhere dense in $A$. 
Proof. Suppose, to the contrary, that there is an open set $U_{0}$ such that $U_{0} \cap A \subset K(A)$. We may assume that cl $U_{0}$ is compact. Using (i) and (ii) above, we can construct sequences $\left\{b_{i}\right\},\left\{U_{i}\right\}$, and $\left\{r_{i}\right\}$ such that

(a) $b_{i} \in A, b_{i} \subset U_{i} \subset \mathrm{cl} U_{i} \subset U_{i-1}$;

(b) $\operatorname{diam} U_{i}<1 / i$;

(c) $H_{k}\left(F_{b_{i+1}}\right) \rightarrow H_{k} \tilde{U}_{i}$ is not monic; and

(d) there is a factorization

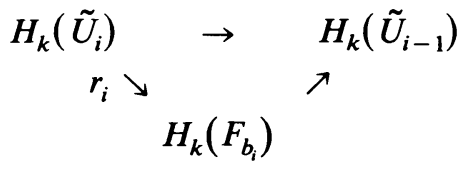

Let $b=\bigcap_{i=1}^{\infty} U_{i}$. Choose an $i$ such that $H_{k}\left(F_{b}\right) \rightarrow H_{k}\left(\tilde{U}_{i}\right)$ is monic. By our choices, the following diagram commutes.

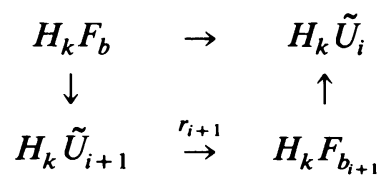

Since $H_{k} F_{b}$ and $H_{k} F_{b_{i+1}}$ have the same rank and $H_{k} F_{b_{i+1}} \rightarrow H_{k} \tilde{U}_{i}$ is not monic, we have a contradiction.

In particular, we shall denote $K(B)$ and $K(K(B))$ by $K$ and $K^{2}$, respectively.

Now, given $b \in B$, let $U$ and $V$ be neighborhoods of $b$ that satisfy (i) and (ii) as above. Given $c \in V$, let $\varphi_{c}$ be the composition $H_{k} F_{c} \rightarrow H_{k} \tilde{V} \stackrel{r}{\rightarrow} H_{k} F_{b}$. Define a function $\alpha_{b}: V \rightarrow R$ by $\alpha_{b}(c)=\left|\operatorname{det} \varphi_{c}\right|$. We say that $\alpha_{b}$ is a $k$-winding function of $f$ about $F_{b}$. When the reference point $b$ is clear from the context, we shall write $\alpha$ for $\alpha_{b}$.

LEMMA 3.2. Let $\alpha_{b}$ be $a k$-winding function for $f$ about $F_{b}$ with domain $V$ and suppose $y \in V$. Then there is a neighborhood $V_{y}$ of $y$ in $V$ and $a k$-winding function $\alpha_{y}$ about $F_{y}$ such that for each $x \in V_{y}, \alpha_{b}(x)=\alpha_{b}(y) \cdot \alpha_{y}(x)$.

Proof. Using (ii), choose $V_{y}$ such that the inclusion $H_{k} \tilde{V}_{y} \rightarrow H_{k} \tilde{V}$ factors through $H_{k} F_{y}$ via $r^{\prime}$. Then in the composition

$$
H_{k} F_{x} \stackrel{i}{\rightarrow} H_{k} \tilde{V}_{y} \stackrel{r^{\prime}}{\rightarrow} H_{k} F_{y} \stackrel{j}{\rightarrow} H_{k} \stackrel{\stackrel{r}{V}}{\rightarrow} H_{k} F_{b}
$$

we have $\alpha_{b}(x)=|\operatorname{det} r j| \cdot\left|\operatorname{det} r^{\prime} i\right|=\alpha_{b}(v) \cdot \alpha_{y}(x)$.

LEMMA 3.3. If $\alpha_{b}: V \rightarrow R$ is a $k$-winding function, then $\alpha_{b}$ is lower semicontinuous at each point of $V-K$.

Proof. If $y \in V-K$, we can choose a neighborhood $V_{y}$ as in the above lemma so that $\alpha_{y}(x) \neq 0$ for all $x \in V_{y}$. Then for $x \in V_{y}, \alpha_{b}(x)=\alpha_{b}(y) \alpha_{y}(x) \geqslant \alpha_{b}(y)$.

LEMMA 3.4. If $\alpha_{b}: V \rightarrow Z$ is a $k$-winding function, the set of points at which $\alpha_{b}$ is continuous is dense and open in $V$.

The proof is a standard argument with lower semicontinuity. See [Bo, p. 395]. 
The next lemma explains why we are interested in the analysis of winding functions.

LEMMA 3.5. For $a$ point $b \in B-K$, the following are equivalent.

(i) Whenever $b$ is in the domain of a $k$-winding function $\alpha_{y}, \alpha_{y}$ is continuous at $b$;

(ii) $\alpha_{b}$ is continuous at $b$; and

(iii) $f$ is $H_{k}$-movable at $b$.

Furthermore, the set of points which satisfy (i)-(iii) is dense and open in $B$.

Proof. Clearly (i) $\Rightarrow$ (ii). Suppose (ii) holds. Given a neighborhood $U_{0}$ of $b$, choose neighborhoods $U$ and $V$ such that $H_{k}\left(F_{b}\right) \rightarrow H_{k}(\tilde{U})$ is monic, and so that $\alpha_{b}$ is defined on $V$. Since $\alpha_{b}$ is continuous at $b$, we may assume $\alpha_{b}(c)=1$ for all $c \in V$. It is easy to check that $V$ and $U$ are neighborhoods needed to verify the definition of $H_{k}$-movability at $b$, so (ii) $\Rightarrow$ (iii). If $f$ is $H_{k}$-movable at $b$, it follows that $b$ has a neighborhood $V$ with $\alpha_{b}=1$ on $V$. (i) then follows from Lemma 3.2. The last sentence of the lemma follows from Lemma 3.4.

THeOREM 3.6. Let $f: E \rightarrow B$ be a proper, surjective mapping between $A N R$ 's such that for each $b \in B, F_{b}$ is a FANR. Suppose that for some index $k$, there is an integer $q$ such that $H_{k}\left(F_{b}\right) \cong Z^{q}$ for every $b \in B$. Then there is a dense open set $B_{k} \subset B$ such that $f \mid f^{-1}\left(B_{k}\right)$ is $H_{k}$-movable.

Proof. This follows directly from the above lemma and the fact that $B-K$ is dense and open.

REMARK. If, in the above theorem, we assume instead that $\check{\pi}_{k}\left(F_{b}\right) \cong Z^{q}$ for all $b \in B$, the same program can be repeated to show that $f$ is $\pi_{k}$-movable over a dense open set.

COROllary 3.7. Let $f: E \rightarrow B$ be a proper, surjective, $U V^{1}$ mapping between finite-dimensional ANR's with FANR point inverses. If all the point inverses have the same shape and $H_{*} F_{b}$ is free, then $f$ is an approximate fibration over a dense open set in $B$.

Proof. Let $n=\operatorname{dim} E$. The set $B_{0}=\bigcap_{k=2}^{n} B_{k}$ is dense and open and $f$ is $H_{k}$-movable over $B_{0}, 2 \leqslant k \leqslant n$. By [C-D $]$, $f$ is $\pi_{k}$-movable over $B_{0}$ for all $k$, so $f$ is an approximate fibration over $B_{0}\left[\mathbf{C}-\mathbf{D}_{2}\right]$.

4. The odd-dimensional case. Throughout this section we assume $f: M^{2 k+1} \rightarrow N^{n}$, $n \geqslant k+1$, is a proper, surjective mapping between manifolds (without boundary) with $U V^{1}$, FANR point inverses and is $\pi_{i}$-movable for $i<k-1$. The next lemma summarizes what has been proven about this situation up to this point.

LEMMA 4.1. Assuming that the Euler characteristic $\chi\left(F_{y}\right)$ is constant, then

(1) $H^{j} F_{y}=0$ for all $j \geqslant k+2$ and $y \in N$;

(2) the set $S=\left\{y \in N \mid H^{k+1} F_{y} \neq 0\right\}$ is locally finite;

(3) there is a dense open set $N_{0}$ such that $f$ is an approximate fibration over $N_{0}$; and

(4) for each $y \in N-S$,

$$
H_{k} F_{y} \cong \begin{cases}0 \text { or } Z, & \text { for } n=k+1 \\ 0, & \text { for } n>k+1\end{cases}
$$


Proof. Statements (1) and (2) follow from Lemma 2.1 and Theorem 2.4. If $y \in N-S$, by the Universal Coefficient Theorem [S], $\operatorname{rank}\left(H_{j} F_{y}\right)=0$ if $j>k+$ 1 , and $H_{k} F_{y}$ is free. Also, $H_{i} F_{y} \cong H_{i} F_{z}$ for all $y, z \in N$ and $i<k-1$ by the $\pi_{i}$-movability and Lemma 1.1. By our Euler characteristic assumption, there is an integer $q$ such that $H_{k}\left(F_{y}\right) \simeq Z^{q}$ for $y \in N-S$. By Theorem 3.6 there is a dense open set $N_{0} \subset N-S$ such that $f$ is $H_{k}$-movable over $N_{0}$, so $f$ is an approximate fibration over $N_{0}$ by $\left[\mathbf{C}-\mathbf{D}_{3}\right]$.

Now the arguments of Husch [H] show that for $b \in N_{0}, F_{b} \times T^{i}$ has the shape of a closed $(2 k+1-n+i)$-manifold where $T^{i}$ is the $i$-fold product of circles for some $i$. Then statement (4) follows from the Künneth formula.

Lemma 4.1 shows that if $\chi\left(F_{y}\right)$ is constant, $f$ is an approximate fibration except over a closed, nowhere-dense set $E$. To analyze the set $E$ further, we need the following lemma whose proof is the same as that of Lemma 5 of [C-D $]$.

LEMMA 4.2. Let $\alpha: V \rightarrow R$ be a $k$-winding function for $f$ about some fiber. Suppose there is an arc $A \subset V$ with $\operatorname{Bd} A=\{c, d\}$ such that $A-\{d\} \subset N_{0} \cap A$ and $\alpha(c)=p \alpha(d)$. Then the inclusion $F_{d} \subset \tilde{A}$ is a shape equivalence and $\varphi: H_{k}\left(F_{c}\right) \rightarrow$ $H_{k}(\tilde{A})$ is multiplication by $p$.

THEOREM 4.3. Let $f: M^{2 k+1} \rightarrow N^{n}$ be a proper surjective $U V^{1}$ mapping between manifolds with FANR point inverses. If $f$ is $\pi_{i}$-movable for all $i\langle k-1, n\rangle k+1$ and $\chi\left(F_{y}\right)$ is constant as $y$ varies through $N$, then there is a countable set $E \subset N$ such that $f$ is an approximate fibration over $N-E$.

PRoof. If $H_{k}\left(F_{y}\right) \cong 0$ for $y \in N-S$, it follows from [C-D $]$ that $f$ is an approximate fibration over $N-S$, so we may as well assume that $n=k+1$ and $H_{k}\left(F_{y}\right) \cong Z$ for all $y \in N-S$. Since $S$ is locally finite, we may assume that $S$ is empty and that $N_{0}$ (from Lemma 4.1) is the dense open set of points for which the three equivalent conditions of Lemma 3.5 hold. We can write $N-N_{0}=D=D_{1}$ $\cup D_{2}$, where $D_{1}$ is perfect and $D_{2}$ is countable. We claim that $D_{1}$ is empty. To show this, we assume $D_{1} \neq \varnothing$ and consider two cases.

Case I. Suppose $D_{1}-K \neq \varnothing$. Then $D_{1}-K$ is dense in itself. Choose a point $b \in D_{1}-K$ and let $\alpha_{b}: V \rightarrow R$ be a $k$-winding function, where $V \cap K=\varnothing$ and $\alpha>0$ on $V$. Since $\alpha \mid V \cap D_{1}$ is lower semicontinuous, there is a point $d \in D_{1} \cap V$ such that $\alpha \mid V \cap D_{1}$ is continuous at $d$. Let $W \subset V$ be a Euclidean neighborhood of $d$ and let $B$ be the interior of a tame $n$-cell in $W$ containing $d$. Choose $B$ small enough that $\alpha(d)$ divides $\alpha(y)$ for every $y \in B$ and $\alpha(y)=\alpha(d)$ for every $y \in B \cap$ $D_{1}$. We claim that $\alpha(c)>\alpha(d)$ for some $c \in N_{0} \cap B$. Otherwise, $\alpha$ would be constant on $B \cap\left(N_{0} \cup D_{1}\right)$, say $\alpha(c)=q$. Then for any $d_{2} \in D_{2} \cap B$, we would have $\alpha\left(d_{2}\right) \leqslant q$ by Lemma 3.2 and $\alpha\left(d_{1}\right) \geqslant \alpha(d)=q$, so $\alpha \mid B$ would be constant. This would imply that $\alpha_{d}=1$ on a neighborhood $d$ by Lemma 3.3, so $d \in N_{0}$ by Lemma 3.5 , a contradiction. Since $D_{1}$ is perfect, there is a sequence $\left\{d_{1}^{\prime}, d_{2}^{\prime}, \ldots\right\}$ of distinct points in $D_{1} \cap B$. Choose arcs $A_{i}^{\prime} \subset B$ such that $\mathrm{Bd} A_{i}^{\prime}=\left\{c, d_{i}^{\prime}\right\}, A_{i}^{\prime} \cap A_{j}^{\prime}$ $=\{c\}, i \neq j$, and $A_{i}^{\prime} \cap D_{2}=\varnothing$. Let $d_{i}$ be the point of $A_{i}^{\prime} \cap D_{1}$ closest to $c$ along $A_{i}^{\prime}$ and $A_{i}$ the subarc of $A_{i}^{\prime}$ from $c$ to $d_{i}$. Let $T_{r}=f^{-1}\left(\cup_{i=1}^{r} A_{i}\right)$. It is straightforward, but tedious, to show that $H_{k+1}\left(T_{r}\right) \simeq 0$ and $H_{k}\left(T_{r}\right) \simeq Z \oplus Z_{p}^{r-1}$, where 
$p=\alpha(d) / \alpha(c)$. One uses induction, Lemma 4.2, and the Mayer-Vietoris sequence. It follows from the Universal Coefficient Theorem that $H^{k+1}\left(T_{r}\right) \simeq\left(Z_{p}\right)^{r-1}$. (Note that $T_{r}$ is a FANR by [D-S, Theorem 9.3.4].) But $H^{k+1}(\tilde{B}) \rightarrow H^{k+1}\left(T_{r}\right)$ is epic and $H^{k+1} \tilde{B}$ is finitely generated by Lemmas 2.2 and 2.3. Thus we have a contradiction and $D_{1}$ is empty.

Case II. Suppose $K \supset D_{1}$. Then $K^{2}$ is nowhere dense in $K$, so there is a point $b \in K-K^{2}$. Let $\alpha_{b}: V \rightarrow R$ be a $k$-winding function such that $V \cap K^{2}=\varnothing$. By the definition of $K$ and $K^{2}$ there is a point $c \in V-K$ such that $\alpha_{b}(c)=0$. By 3.2, we may assume that $c \in V-D$. Also, since $b \notin K^{2}$, we may assume that $\alpha_{b}(x)>0$ for $x \in K \cap V$. Let $\left\{d_{i}^{\prime}\right\}$ be a sequence of distinct points in $K \cap V$ and choose $\left\{A_{i}^{\prime}\right\},\left\{A_{i}\right\}$ and $\left\{d_{i}\right\}$ as in Case I. It follows from Lemma 4.2 that $H_{k}\left(F_{c}\right) \rightarrow H_{k}\left(\tilde{A}_{i}\right)$ is the zero map. As in Case I, let $T_{r}=f^{-1}\left(\cup_{i=1}^{r} A_{i}\right)$. It follows that $H_{k+1}\left(T_{r}\right) \cong Z^{r-1}$, so that $H^{k+1}\left(T_{r}\right)$ can be generated by no fewer than $r-1$ elements. This is a contradiction as in Case I. Thus $D=N-N_{0}$ is at most countable.

ADDENDUM. The set $D_{2}$ is locally finite in $N-S$.

Proof. Suppose there is a point $d_{0} \in D_{2}$, every neighborhood of which contains infinitely many points of $D_{2}$. Let $\alpha: V \rightarrow R$ be a $k$-winding function for $f$ around some point inverse where $d_{0} \in V$. Lemma 3.2 implies that for every $d \in D_{2} \cap V$ there is a $c \in N_{0} \cap V$ such that $\alpha(d) \leqslant \alpha(c)$. But $\alpha$ is constant on $N_{0} \cap V$ so $\alpha$ is bounded on $V$. Hence there is a sequence $\left\{d_{i}\right\}$ converging to $d_{0}$ such that $\alpha\left(d_{i}\right)=p \alpha(c)$ for every $i$ and some fixed $p \neq 1$. This yields a contradiction as in the previous proof.

THEOREM 4.4. Let $f: M^{2 k+1} \rightarrow N^{n}$ be a $U V^{1}$ mapping between manifolds with FANR point inverses, all of which have the same shape. If $f$ is $\pi_{i}$-movable for all $i \leqslant k-1, n \geqslant k+1$, there is a locally finite set $E \subset N$ such that $f$ is an approximate fibration over $N-E$.

5. $k$-sphere mappings. We now turn to the special case of a mapping $f: S^{2 k+1} \rightarrow$ $S^{k+1}$ with the property that each point inverse has the shape of $S^{k}$. This topic was studied in two of the antecedents $\left[\mathbf{C}-\mathbf{D}_{\mathbf{3}}\right],\left[\mathbf{C}-\mathbf{D}_{\mathbf{4}}\right]$ of this paper. However, one loose end was left which we now tie up. Specifically, the earlier papers assumed that the mapping was nondegenerate; i.e., $K=\varnothing$. As a result of Lemma 3.1 we can now extend the earlier work to cover the case $K \neq \varnothing$ as well.

THEOREM 5.1. If $f: S^{2 k+1} \rightarrow S^{k+1}, k \geqslant 1$, is a $k$-sphere mapping, then $f$ is an approximate fibration over the complement of a finite set, $D \cup K$. If $K=\varnothing$, then $D$ has no more than two points if $k=1$, and no more points than the number of prime factors of the Hopf invariant of $f$ if $k \geqslant 2$. If $K \neq \varnothing$, then $D=\varnothing$ and $K$ is $a$ singleton.

Proof. For $k \geqslant 2$, the first conclusion follows from Theorem 4.3 and its addendum since $S=\varnothing$. For $k=1$, one can apply the proof of Theorem 4.3 using $\pi_{1}$ in place of $H_{k}$ to obtain the first conclusion. The second conclusion follows 
directly from $\left[\mathbf{C}-\mathbf{D}_{3}\right]$ and $\left[\mathbf{C}-\mathbf{D}_{4}\right]$. To prove the last statement, let $a \in K, b \in D \cup$ $K$ be distinct points and let $A$ be an arc between $a$ and $b$ such that $A-\{a, b\} \subset$ $S^{k+1}-(D \cup K)$. Letting $\alpha$ be a $k$-winding function about $b$, choose a point $c \in$ int $A$ such that $\alpha(c)=0$ if $b \in K$ or $\alpha(c)=p>1$ if $b \in D$. Let $c$ divide $A$ into subarcs $A_{a}$ and $A_{b}$ with $a \in A_{a}$ and $b \in A_{b}$. Then from Lemma 4.2 we have that $H_{k}\left(F_{c}\right) \rightarrow H_{k}\left(\tilde{A}_{a}\right)$ is the zero homomorphism and $H_{k}\left(F_{c}\right) \rightarrow H_{k}\left(\tilde{A}_{b}\right)$ is multiplication by $p$ (or zero). It follows from a Mayer-Vietoris argument that $H^{k+1}(\tilde{A}) \cong Z$ or $Z_{p}$. On the other hand,

$$
H^{k+1} \tilde{A} \cong H_{k-1}\left(S^{2 k+1}-\tilde{A}\right) \cong H_{k-1}\left(S^{k+1}-A\right) \cong H^{1}(A) \cong 0,
$$

where the first and third isomorphisms are from duality and the second from the fact that $f$ is a $U V^{k-1} \operatorname{map}\left[\mathbf{L}_{3}\right]$. This contradiction completes the proof.

6. Examples. In this section we give several examples of mappings which show that some of the hypotheses of Theorems 2.5 and 4.4 are necessary.

EXAMPLE 6.1. For any $k \geqslant 2$, there is a $U V^{1}$ mapping $p_{1}: E \rightarrow N$ where $E$ is a $(2 k+1)$-dimensional ANR, $N$ is a $(k+1)$-manifold, $p_{1}$ is $\pi_{i}$-movable for $i \leqslant k-$ 1 , and all fibers have the same shape, but the set of points in $N$ where $\pi_{k}$-movability fails is not locally finite.

Proof. Let $\nu: S^{1} \times S^{k} \rightarrow S^{k+1}$ be the map which identifies the "spine" $\left(S^{1} \times\right.$ $\{y\}) \cup\left(\{z\} \times S^{k}\right), y \in S^{k}, z \in S^{1}$ fixed, to the point $n=(0,0, \ldots, 0,1)$ in $S^{k+1}$ and is a homeomorphism elsewhere. Next let $\mu: S^{k+1} \rightarrow[-1,1]$ be defined by $\mu\left(x_{1}, \ldots, x_{k+2}\right)=x_{k+2}$. Then $\phi=\mu \circ \nu: S^{1} \times S^{k} \rightarrow[-1,1]$ satisfies

$$
\phi^{-1}(t) \cong \begin{cases}\text { point } & \text { if } y=-1, \\ S^{k} & \text { if }-1<t<1, \\ S^{1} \vee S^{k} & \text { if } t=1 .\end{cases}
$$

Let $\bar{\phi}: S^{1} \times S^{k} / L \rightarrow[-1,1]$ be induced by $\phi$, where $L=S^{1} \times\{y\}$. Define $E_{1}=\bar{\phi}^{-1}([0,1])$ and let $f_{1}=\bar{\phi} \mid E_{1}$. Then $f_{1}^{-1}(t) \simeq S^{k}$ for each $t \in[0,1]$ but $f_{1}$ is not $\pi_{k}$-movable at $t=1$.

Similarly, for each $i$, there is a mapping $f_{j}: E_{j} \rightarrow[j-1, j]$ where $E_{j}$ is a copy of $E_{1}$ disjoint from $E_{l}, j \neq l, f_{j}$ is defined the same way as $f_{1}$. Let $E_{\infty}=\cup_{j=1}^{\infty} E_{j}$ with $f_{j}^{-1}(j)$ identified to $f_{j+1}^{-1}(j)$ by a homeomorphism. Now take the one point compactification of $E_{\infty}$ and identify the point at infinity with some point on $f_{1}^{-1}(0)$ to get the space $E^{\prime}$. Now define $f: E^{\prime} \rightarrow S^{1}$ by $f(x)=\exp 2 \pi i\left[f_{j}(x) /\left(1+f_{j}(x)\right)\right]$ when $x \in E_{j}$ and $f(\infty)=1$. Finally let $E=E^{\prime} \times B^{k}, N=S^{1} \times B^{k}$, and $p_{1}=f \times$ id. Then each point inverse of $p_{1}$ is a $k$-sphere, but $p_{1}$ fails to be $\pi_{k}$-movable at each $(x, y)$ where $x=\exp 2 \pi i(n /(n+1))$ for some nonnegative integer $n$ and $y \in B^{k}$.

EXAMPLE 6.2. There is a $U V^{1}$ map $p_{2}: S^{2 k+1} \rightarrow S^{k+1}, k \geqslant 3$, such that $p_{2}$ is $\pi_{i}$-movable for $i \leqslant k-1$ and each point inverse is an FANR, but $p_{2}$ fails to be $k$-movable along an arc.

Proof. By [C, Corollary 13] there is a map $f: S^{2 k+1} \rightarrow S^{2 k+1}$ such that one point inverse is a $k$-sphere, the other nondegenerate point inverses are $(k-1)$-connected polyhedra, and the image of the nondegenerate point inverses is a tame $\operatorname{arc} A$. Let $g: S^{2 k+1} \rightarrow S^{k+1}$ be a $k$-sphere mapping $\left[\mathbf{L}_{\mathbf{4}}\right],\left[\mathbf{C}-\mathbf{D}_{\mathbf{4}}\right],[\mathbf{F}-\mathbf{W}]$. We may assume that 
$A$ intersects each fiber of $g$ in a point or the empty set. Then $p_{2}=g \circ f$ is the desired map. Note that $p_{2}$ is not $k$-movable along $g(A)$.

EXAMPLE 6.3. For $k \geqslant 2$ there is a $U V^{1}$ map $p_{3}: S^{k} \times S^{k+1} \rightarrow S^{k+1}$ such that $p_{3}$ is $\pi_{i}$-movable for $i \leqslant k-1$ and all the point inverses have the same shape, but $p_{3}$ fails to be $k$-movable everywhere.

LeMma 6.4. For $n \geqslant 1$, there exists a surjection $f: I \rightarrow B^{n}$ such that every point inverse is a Cantor set and $f(0) \in \mathrm{Bd} B^{n}$.

Proof. Let $f: R \rightarrow R$ be defined by $g(x)=m x$ where $m \neq 0$. For any $a>0$, define the standard zig-zag of $g$ on $[0, a]$ to be the function $g^{\prime}$ defined by

$$
g^{\prime}(x)= \begin{cases}2 m x & \text { if } 0 \leqslant x \leqslant a / 4, \\ -2 m x+m a & \text { if } a / 4<x<a / 2, \\ 2 m x-m a & \text { if } a / 2<x<a, \\ g(x) & \text { if } x \leqslant 0 \text { or } x>a .\end{cases}
$$

If $h$ is any function which is affine on $[b, c]$, define a zig-zag of $h$ on $[b, c]$ by

$$
h^{\prime}(x)= \begin{cases}g^{\prime}(x-b)+h(b) & \text { if } b \leqslant x<c, \\ h(x) & \text { if } x<b \text { or } x \geqslant c,\end{cases}
$$

where $g^{\prime}$ is the standard zig-zag of $g(x)=h(x+b)-h(b)$ on $[0, c-b]$.

Now define $f_{1}:[0,1] \rightarrow[0,1]$ by

$$
f_{1}(x)= \begin{cases}2 x & \text { if } 0 \leqslant x<\frac{1}{2}, \\ 2-2 x & \text { if } \frac{1}{2} \leqslant x \leqslant 1 .\end{cases}
$$

Let $f_{2}$ be obtained by applying a zig-zag to $f_{1}$ on each of the intervals $\left[0, \frac{1}{2}\right]$ and $\left[\frac{1}{2}, 1\right]$. Then define $f_{3}$ by applying a zig-zag to $f_{2}$ on each of the intervals $\left[0, \frac{1}{8}\right]$, $\left[\frac{1}{8}, \frac{1}{4}\right], \ldots,\left[\frac{7}{8}, 1\right]$. Continue dividing each interval from the previous stage into four subintervals and zig-zagging on each one to produce the next stage. The sequence $\left\{f_{i}\right\}$ converges uniformly to a continuous function $f$ since $d\left(f_{i}, f_{i+1}\right)<2^{-i}$. Each $f^{-1}(y)$ can be seen to be a Cantor set as follows. First

$$
f^{-1}(y)=\bigcap_{i=1}^{\infty} f_{i}^{-1}\left(y-2^{-i+1}, y+2^{-i+1}\right) .
$$

Secondly each $f_{i}^{-1}\left(y-2^{-i+1}, y+2^{-i+1}\right)$ consists of $2^{i}$ intervals, of which two lie in each interval of $f_{i-1}^{-1}\left(y-2^{-i+2}, y+2^{-i+2}\right)$ provided both $|y|$ and $|y-1|$ are greater than $2^{-i+2}$. This shows that $f^{-1}(y)$ is a Cantor set whenever $y \neq 0,1$. Those two point inverses are intersections of sequences of somewhat fewer intervals at each stage but nevertheless can be seen to be Cantor sets by inspection. To get such mappings onto $B^{n}, n>1$, compose $f$ with any mapping onto $B^{n}$ such that the point inverses are totally disconnected and 0 goes to $\mathrm{Bd} B^{n}$.

Lemma 6.5. For $k \geqslant 2$ there exists an embedding $F:[-1,1] \rightarrow S^{k} \times S^{k+1}$ such that $A=F[-1,1]$ is a tame arc and $A \cap \pi_{2}^{-1}(y)$ is a Cantor set for every $y$ in $S^{k+1}$. 
Proof. Write $S^{k+1}=U \cup L$, the union of the upper and lower hemispheres. Let $h_{U}: B^{k+1} \rightarrow U$ and $h_{L}: B^{k+1} \rightarrow L$ be homeomorphisms with $h_{L}\left|S^{k}=h_{U}\right| S^{k}$. Let $g:[-1,1] \rightarrow S^{k}$ be an embedding. Now define $F:[-1,1] \rightarrow S^{k} \times S^{k+1}$ by

$$
F(t)= \begin{cases}\left(g(t), h_{U} f(t)\right) & \text { if } 0 \leqslant t \leqslant 1, \\ \left(g(t), h_{L} f(-t)\right) & \text { if }-1<t<0,\end{cases}
$$

where $f:[0,1] \rightarrow B^{k+1}$ is the function described in Lemma 6.4. Clearly $F$ is an embedding and $A=F([-1,1])$ intersects $S^{k} \times\{y\}$ in a Cantor set for every $y \in S^{k+1}$. Also $A$ is tame since $A \cap\left(\{Z\} \times S^{k+1}\right)$ is a single point or the empty set.

Proof of Example 6.3. There exists a $U V^{k-1}$ mapping $f_{1}: B^{2 k+1} \rightarrow B^{2 k+1}$ such that $f_{1}=$ id on $B d B^{2 k+1}$, two point inverses are $k$-spheres, the other nondegenerate point inverses are wedges of two $k$-spheres, all of which fit together concentrically to form two $(k+1)$-cells meeting along a radius of each. (The 3-dimensional and original example is Bing's "circles and figure eights" decomposition of $S^{3}$ [Bi].) Now consider $B^{2 k+1}$ to be a $(2 k+1)$-cell in $S^{k} \times S^{k+1}$ and extend $f_{1}$ to $f_{2}$ : $S^{k} \times S^{k+1} \rightarrow S^{k} \times S^{k+1}$ via the identity outside $B^{2 k+1}$. Since the image of the nondegenerate point inverses of $f_{2}$ is a tame arc, it can be assumed to be the $\operatorname{arc} A$ of Lemma 6.5. Finally, define $p_{3}: S^{k} \times S^{k+1} \rightarrow S^{k+1}$ by $p_{3}=\pi_{2} \circ f_{2}$. First, $p_{3}$ is $\pi_{k-1}$-movable, since for any nested pair $C_{1} \subset C_{2}$ of $2 k$-cells in $S^{k+1}, p_{3}^{-1}\left(C_{1}\right) \subset$ $p_{3}^{-1}\left(C_{2}\right)$ is a $(k-1)$-equivalence [C-D $\mathbf{D}_{5}$. Secondly, each point inverse has the shape of a wedge of an infinite collection of $k$-spheres. To see this, notice that $\pi_{2}^{-1}(y)$ is a $k$-sphere which intersects $A$ in a Cantor set. Then $p_{3}^{-1}(y)$ is a $k$-sphere with either a $k$-sphere or a wedge of two $k$-spheres attached to it at each point of the Cantor set. By [Sp] this has the shape of an infinite wedge of $k$-spheres. Finally, $p_{3}$ cannot be an approximate fibration over any open set since $p_{3}^{-1}(y)$ is not a FANR, but the fibers of an approximate fibration between ANR's are FANR's [C-D ] .

EXAMPLE 6.6. The $U V^{1}$ degree 2 map $H: S^{5} \rightarrow S^{5}$ constructed by Wilson [W, Theorem 2] satisfies the hypotheses of Theorem 4.4 except that the point inverses are not FANR's with the same shape. However, $H$ is not an approximate fibration over any open set since otherwise $H$ would be cell-like on that open set by [D-H]. Cell-like maps are hereditary homotopy equivalences [ $\mathbf{L}_{\mathbf{3}}$, Theorem 4.2] which cannot have degree 2.

EXAMPLE 6.7. The mappings which Lacher calls spine maps $\left[\mathbf{L}_{5}\right.$, p. 519$]$ can be used to construct several relevant examples. Roughly a mapping $s: M \rightarrow Q$ between two $m$-manifolds is a spine map if $M \cong Q \# K_{1} \# \ldots \# K_{i} \# \ldots$ (\#) denotes connected sum) and $s$ crushes a spine of each $K_{i}$. If each $K_{i}$ is $(k-1)$ connected, then $s$ is $U V^{k-1}$. The composition $f=p \circ s$ of a $U V^{k-1}$ spine map and a fibration $p: Q^{m} \rightarrow N^{k+1}, m=2 k$, shows that Theorem 2.5 cannot be improved to conclude that $f$ is an approximate fibration everywhere. The same kind of example in the odd-dimensional case, $m=2 k+1$, shows that the set $S$ in Lemma 4.1 can be nonempty. Furthermore, the composition

$$
\left(S^{k} \times S^{k+1}\right) \# S^{2 k+1} \rightarrow S^{2 k+1} \rightarrow S^{k+1}
$$

of a spine map and a $k$-sphere mapping is an example in which the fibers have 
constant Euler characteristic, but which is not an approximate fibration everywhere. (Compare this to Theorem 4.3.) Of course a $k$-sphere mapping alone shows that Theorem 4.4 cannot be improved to conclude that the map is an approximate fibration everywhere.

On the other hand if $s: M \rightarrow Q$ is a $U V^{k-2}$ spine map on $(2 k-2)$-manifolds which is not $U V^{k-1}$ and $p: Q \rightarrow N^{k-1}$ is any fibration, then

$$
M \times S^{2} \stackrel{s \times 1}{\rightarrow} Q \times S^{2} \stackrel{p \times 1}{\rightarrow} N \times S^{2}
$$

shows that $\pi_{i}$-movability for $i \leqslant k-2$ does not suffice in Theorem 2.5. Similarly the composition

$$
\left[\left(S^{k} \times S^{k-1}\right) \#\left(S^{k} \times S^{k-1}\right)\right] \times S^{2} \rightarrow\left[S^{k} \times S^{k-1}\right] \times S^{2} \rightarrow S^{k-1} \times S^{2}
$$

of a $U V^{k-2}$ spine map cross $1_{S^{2}}$ with a projection shows that $\pi_{i}$-movability for $i \leqslant k-2$ does not suffice in Theorem 4.3.

\section{BIBLIOGRAPHY}

[B] K. Borsuk, $A$ note on the theory of shape of compacta, Fund. Math. 67 (1970), 265-278.

[Bi] R. H. Bing, Decompositions of $E^{3}$, Topology of 3-Manifolds and Related Topics, Prentice-Hall, Englewood Cliffs, N. J., 1962.

[Bo] N. Bourbaki, Elements of mathematics: General topology, Addison-Wesley, Reading, Mass., 1966.

[C] D. Coram, Semicellularity, decompositions, and mappings in manifolds, Trans. Amer. Math. Soc. 191 (1974), 227-244.

[C-D $]$ D. Coram and P. Duvall, Approximate fibrations, Rocky Mountain J. Math. 7 (1977), 275-288.

$\left[\mathrm{C}-\mathrm{D}_{2}\right]$ _ Approximate fibrations and a movability condition for maps, Pacific J. Math. 72 (1977), 41-56.

[C-D $\left.\mathbf{D}_{3}\right]$ , Mappings from $S^{3}$ to $S^{2}$ whose point inverses have the shape of a circle, General Topology Appl. 10 (1979), 239-246.

[C-D $\mathrm{D}_{4}$ _ Non-degenerate $k$-sphere mappings, Topology Proc. 4(1979), 67-82.

[C-D $\left.\mathrm{D}_{5}\right]$, A Hurewicz-type theorem for approximate fibrations, Proc. Amer. Math. Soc. 78 (1980), 443-448.

[D-H] P. F. Duvall, Jr. and L. S. Husch, Fundamental dimension of fibers of approximate fibrations, Topology Proc. 3 (1978), 53-57.

[D-S] J. Dydak and J. Segal, Shape theory, Lecture Notes in Math., vol. 688, Springer-Verlag, New York, 1978.

[F-W] R. Fintushel and J. J. Walsh, Singularly fibered homotopy spheres (to appear).

[H] L. S. Husch, Fibers of Hurewicz and approximate fibrations, Math. Scand. 43 (1978), 44-48.

[L $\mathbf{L}_{1}$ ] R. C. Lacher, Cellularity criteria for maps, Michigan Math. J. 17 (1970), 385-396.

$\left[\mathbf{L}_{2}\right]$, Finiteness theorems in the study of mappings between manifolds, Proc. Univ. Oklahoma Topology Conf. (1972), Univ. of Oklahoma, Norman, pp. 79-96.

$\left[\mathbf{L}_{3}\right]$ _ Sorne mapping theorems, Trans. Amer. Math. Soc. 195 (1974), 291-303.

$\left[L_{4}\right], k$-sphere mappings on $S^{2 k+1}$, Proc. Utah Geometry Topology Conf. 1974 (L. C. Glaser and T. B. Rushing, editors), Lecture Notes in Math., vol. 438, Springer-Verlag, New York and Berlin, 1975.

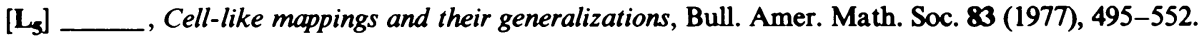

[L-M] R. C. Lacher and D. R. McMillian, Partially acyclic mappings between manifolds, Amer. J. Math. 94 (1972), 246-266.

[M] S. Mardesic, Strongly movable compacta and shape retracts, Proc. Internat. Sympos. Topology Appl. (Budva, 1972), Savez Društava Mat. Fiz. Astronom., Beograd, 1973, pp. 163-166.

[O] R. Overton, Čech homology for movable compacta, Fund. Math. 77 (1973), 241-251.

[S] E. H. Spanier, Algebraic topology, McGraw-Hill, New York, 1966.

[Sp] S. Spiez, On a characterization of shapes of several compacta, Bull. Acad. Polon Sci. Sér. Sci. Math. 24 (1976), 257-263.

[W] D. C. Wilson, On constructing monotone and $U V^{1}$ mappings of arbitrary degree, Duke Math. J. 41 (1974), 103-109. 